\title{
Ambulatory surgery with chloroprocaine spinal anesthesia: a review
}

This article was published in the following Dove Press journal:

Ambulatory Anesthesia

2 November 2015

Number of times this article has been viewed

\author{
Daniela Ghisi \\ Stefano Bonarelli \\ Department of Anaesthesia and \\ Postoperative Intensive Care, Istituto \\ Ortopedico Rizzoli, Bologna, Italy
}

\begin{abstract}
Spinal anesthesia is a reliable and safe technique for procedures of the lower extremities. Nevertheless, some of its characteristics may limit its use for ambulatory surgery, including delayed ambulation, risk of urinary retention, and pain after block regression. The current availability of short-acting local anesthetics has renewed interest for this technique also in the context of short- and ultra-short procedures. Chloroprocaine (CP) is an amino-ester local anesthetic with a very short half-life. It was introduced and has been successfully used for spinal anesthesia since 1952. Sodium bisulfite was then added as a preservative after 1956 . The drug was then abandoned in the 1980s for several reports of neurological deficits in patients receiving accidentally high doses of intrathecal CP during epidural labor analgesia. Animal studies have proven the safety of the preservative-free formulation, which has been extensively evaluated in volunteer studies as well as in clinical practice with a favorable profile in terms of both safety and efficacy. In comparison with bupivacaine, 2-chloroprocaine (2-CP) showed faster offset times to end of anesthesia, unassisted ambulation, and discharge from hospital. These findings suggests that 2-CP may be a suitable alternative to low doses of long-acting local anesthetics in ambulatory surgery. Its safety profile also suggests that 2-CP could be a valid substitute for intrathecal short- and intermediate-acting local anesthetics, such as lidocaine and mepivacaine often causes of transient neurological symptoms. In this context, literature suggests a dose ranging between 30 and $60 \mathrm{mg}$ of 2-CP for procedures lasting 60 minutes or less, while $10 \mathrm{mg}$ is considered the no-effect dose. The present review describes recent evidence about 2-CP as an anesthetic agent for spinal anesthesia in ambulatory surgery.
\end{abstract}

Keywords: outpatient surgery, spinal anesthesia, 2-chloroprocaine

\section{Introduction}

In the last years, the number of surgical procedures performed on an ambulatory basis has increased worldwide: ${ }^{1}$ between $50 \%$ and $70 \%$ of all surgeries are currently performed as outpatient procedures in North America alone. ${ }^{2}$

Spinal anesthesia is a safe and reliable technique for surgery of the lower abdomen and lower limbs. ${ }^{3,4}$ Nevertheless, some of its characteristics may limit its use for ambulatory surgery, including delayed ambulation, risk of urinary retention, and pain after block regression. ${ }^{5}$ The choice of the correct local anesthetic for spinal anesthesia is therefore crucial in the ambulatory setting: the ideal anesthetic should allow rapid onset and offset of its own effect for fast patient discharge with minimal side effects. ${ }^{6}$

In the past, the lack of the ideal spinal local anesthetic and the availability of fast-acting drugs such as remifentanil and propofol have made general anesthesia the preferred choice for short outpatient procedures. ${ }^{7,8}$ To investigate the most suitable anesthetic technique for day surgery, Liu et al published a meta-analysis in 2005
Correspondence: Daniela Ghisi Department of Anaesthesia and Postoperative Intensive Care, Istituto Ortopedico Rizzoli, via GC Pupilli, I - 40 I 36 Bologna, Italy

Tel +39340265334I

Fax +3905I 6366356

Email ghisidan@hotmail.com submit your manuscript | www.dovepress.com

Dovepress

http://dx.doi.org// 0.2147/AA.S64884
Ambulatory Anesthesia 2015:2 III-120

(c) (1) (5) 2015 Ghisi and Bonarelli. This work is published by Dove Medical Press Limited, and licensed under Creative Commons Attribution - Non Commercial (unported, v3.0) License. The full terms of the License are available at http://creativecommons.org/licenses/by-n/3.0/. Non-commercial uses of the work are permitted without any further permission from Dove Medical Press Limited, provided the work is properly attributed. Permissions beyond the scope of the License are administered by Dove Medical Press Limited. Information on
how to request permission may be found at: http://www.dovepress.com/permissions.php 
comparing regional and general anesthesia, including more than 1,300 patients. ${ }^{9}$ Regional anesthesia reduced pain scores and pain medication request in the post-anesthesia care unit. However, neither central neuraxial block nor peripheral nerve blocks decreased the overall ambulatory surgery unit time and both required longer induction time versus general anesthesia. ${ }^{9}$ However, the majority of studies included in the meta-analysis of Liu et al used long-acting or intermediate-acting local anesthetics for regional anesthesia, which may have delayed fulfillment of discharge criteria. Although low doses of long-acting local anesthetics such as bupivacaine, ropivacaine, and levobupivacaine are usually administered intrathecally, they are associated with significant risk of delays in hospital discharge and less reliability of block efficacy, onset, and spread. ${ }^{10}$

Short-acting local anesthetics may therefore represent a valid alternative in this setting. Lidocaine has been the anesthetic of choice for years in the context of outpatient procedures. Nevertheless, its use has been associated with a significant risk of transient neurological symptoms (TNS) and most anesthesiologists have therefore abandoned its use. ${ }^{11,12}$ Mepivacaine has been associated as well with transient neurological symptoms. ${ }^{12}$

The recent re-introduction of intrathecal articaine, chloroprocaine $(\mathrm{CP})$, and prilocaine may offer a solution in the ambulatory setting, with a slightly faster profile for CP. ${ }^{13}$

\section{CP}

$\mathrm{CP}$ is an amino-ester local anesthetic with a very short halflife. ${ }^{7}$ It was introduced and has been successfully used for spinal anesthesia since $1952 .{ }^{14}$ Sodium bisulfite was then added as a preservative after 1956 to the commercially available CP preparation, named Nesacaine-caudal and epidural. The drug was used as an epidural anesthetic for obstetric patients. In the early 1980s, several reports of neurologic deficits possibly associated with inadvertent intrathecal injection of large volumes of CP during labor analgesia were published. ${ }^{15-18}$

Animal studies showed irreversible block after exposure of rabbit nerves to Nesacaine-caudal and epidural. ${ }^{19}$ Since a solution of $2 \mathrm{mg} / \mathrm{mL}$ sodium bisulfite and low $\mathrm{pH}$ without $\mathrm{CP}$ similarly led to irreversible block only at low $\mathrm{pH}$, the preservative sodium bisulfite was often considered to be responsible for neural damage in an acidic environment. ${ }^{19}$ Nevertheless, in another study, rats developed more severe injuries after $\mathrm{CP}$ alone through intrathecal catheters than after CP with $2 \mathrm{mg} / \mathrm{mL}$ sodium bisulfite. Moreover, in the same study, the intrathecal administration of bisulfite alone was comparable to normal saline in rats. ${ }^{20}$ Although the reason of such divergent findings is not clear, a key role was probably played not only by different relative dosing of CP and bisulfite, but especially by the different susceptibility of different model systems to sulfur dioxide. The latter comes from the evidence that different levels/activity of sulfite oxidase (the protective enzyme that catalyzes the conversion of sulfites to the less toxic sulfates) exist in mammalian tissues. ${ }^{21,22}$

All preservatives and antioxidants have been removed from two of the three currently available preparations of $\mathrm{CP}$. In Europe, preservative-free 2-chloroprocaine (2-CP) is available as a $10 \mathrm{mg} / \mathrm{mL}$ solution (Ampres, Sintetica, Mendrisio, Switzerland), which was recently approved by the European Medicine Agency for intrathecal use, while it is currently available in the United States as a bisulfite-free solution (Nesacaine-MPF ${ }^{\circledR}$ from Astra Pharmaceuticals, Wilmington, DE, USA; generic CP from Bedford Pharmaceuticals, Bedford, OH, USA) as well as with preservative, although at a lower dose (generic CP from Abbott Laboratories, Abbott Park, IL, USA; sodium bisulfite $=1.8 \mathrm{mg} / \mathrm{mL}$ versus 2.0 $\mathrm{mg} / \mathrm{mL}$ of the original preparation). Due to availability of preservative-free solutions and since human studies have been conducted with the bisulfite-free $\mathrm{CP}$, the bisulfite containing formulation is not indicated for intrathecal administration.

\section{Spinal use of 2-CP}

The use of preservative-free 2-CP for spinal anesthesia has been studied both in healthy volunteers and in patients. ${ }^{23-28}$ Main results of randomized controlled trials about the intrathecal use of CP published between 2004 and 2015 are reported in Table 1.

Besides volunteer studies, a chart review at the Virginia Mason Medical Center, published in 2004, evaluated the first 122 patients receiving spinal anesthesia with 2-CP: in terms of safety, the authors did not find any transient neurological symptom nor any sign of neurotoxicity. ${ }^{29}$ Four patients required general anesthesia to complete surgery because of block resolution during the procedure, while two out of three patients with combined spinal/epidural anesthesia required epidural doses due to surgical length beyond the scheduled time. The 116 remaining patients tolerated the duration of surgery ( $\leq 60$ minutes) with adequate surgical anesthesia and no complications. ${ }^{29}$ This clinical retrospective analysis confirms also data about CP efficacy from volunteer studies. ${ }^{23-28}$ The majority of patients received a dose of $40 \mathrm{mg}$ of 2-CP, which was also the most common dose in preclinical trials. Time intervals until ambulation for 30 and $40 \mathrm{mg}$ of spinal 2-CP were consistent with previous volunteer studies, while 
time to hospital discharge was slightly longer in surgical patients. $^{29}$

Another review from the same Institution was published in 2011 including 563 patients undergoing 601 ambulatory surgeries with spinal anesthetics between August 2004 and March 2006. ${ }^{30}$ The 601 spinal anesthetics reviewed included 503 (84\%) plain 2-CP and a lower percentage of hyperbaric lidocaine, hyperbaric bupivacaine, hyperbaric procaine, and plain mepivacaine, and safety and efficacy of $\mathrm{CP}$ was confirmed in the ambulatory setting in genitourinary procedures in the vast majority of cases, then orthopedic, general, and gynecologic surgeries. A preservative- and antioxidant-free formulation of 2\% plain 2-CP (AstraZeneca plc, London, UK) was administered. Surgery lasted a mean time of $38 \pm 23$ minutes. Primary failure of spinal block had an overall incidence of $1.2 \%$ due to inadequate spinal anesthesia and a secondary failure of $0.8 \%$ due to unanticipated surgical procedure length requiring conversion to general anesthesia.

Postoperative pain and urinary retention were the main reasons for delayed discharge from hospital in the study by Yoos and Kopacz. ${ }^{29}$ Nevertheless, those five patients showing urinary retention underwent surgeries which increase the risk of urinary retention per se (trans-urethral resection of bladder tumor and perirectal surgery). ${ }^{31}$ Also in the review by Hejtmanek and Pollock, ${ }^{30}$ urinary retention was confirmed as the most common post-anesthesia care unit side effect, although also in this case, $83 \%$ of patients underwent procedures characterized by a higher risk for urinary retention, such as cystoscopy, extracorporeal shock wave lithotripsy, peri-rectal or hernia surgery. ${ }^{32}$ Urinary retention is in fact a possible side effect of spinal block, especially with the use of bupivacaine and/or the addition of epinephrine to the local anesthetic. ${ }^{32}$ Despite an increased risk, Smith et al did not find any difference in the incidence of urinary retention with the addition of epinephrine to 2-CP in their volunteer studies, but $100 \%$ of volunteers reported vague, flulike symptoms with its use - a side effect not previously reported with the addition of epinephrine to other local anesthetics. ${ }^{23}$ Authors hypothesized that these symptoms could be related to the acidic $\mathrm{pH}$ (3.5) of combined 2-CP and epinephrine and possibly to the trace amounts of bisulfite in the epinephrine vials. The use of epinephrine should therefore be avoided in combination with 2-CP for spinal injection. ${ }^{23}$ Although currently debated, voiding has been traditionally considered a prerequisite for home discharge to avoid urinary retention especially after spinal anesthesia performed with long-acting local anesthetics. ${ }^{33}$ However, this can prolong hospital stay unnecessarily. Interestingly, it has been reported that if no surgery-related or underlying risk factors for urinary retention are present and short-acting local anesthetics are administered for neuraxial block the incidence of urinary retention is acceptably low. ${ }^{34}$ Mulroy et al suggested a relaxation of the requirements for voiding before hospital discharge in outpatients receiving spinal block with short-duration drugs and undergoing surgical procedures at low risk of urinary retention, such as lower limb surgery. ${ }^{35}$

Besides urinary retention, another possible complaint after spinal anesthesia is the occurrence of transient neurological symptoms, of which the lithotomy or flexed-knee positions (knee arthroscopy) are independent risk factors. ${ }^{36}$ Nevertheless, in the survey by Yoos and Kopacz there are no case reports of TNS although $50 \%$ of patients were in the lithotomy or flexed-knee positions during surgery and $10 \%$ of cases had surgery in the prone jackknife position. ${ }^{29}$ Also in the following retrospective analysis by Hejtmanek and Pollock, no case of TNS-like symptoms or neurotoxicity was reported and the preservative-free formulation of 2-CP has become the short-acting local anesthetic of choice at Virginia Mason Medical Center as a safe and effective alternative to lidocaine and procaine for short ambulatory procedures. $^{30}$

Many authors investigated the correct spinal dose of 2-CP to assure adequate efficacy and fast resolution of block in the ambulatory setting. Sell and Pitkanen tested four different doses of spinal 2-CP $(35,40,45$, and $50 \mathrm{mg})$ in a cohort of 64 patients scheduled for elective lower extremity procedures. The regression of sensory block and time to discharge were faster in the lower dose groups ( 35 and $40 \mathrm{mg}$ ), although the higher level blocked and time to complete block regression were comparable in all four groups. ${ }^{37}$

In an attempt to find the minimum effective dose for intrathecal injection, Kopacz tested 10 and $20 \mathrm{mg}$ of plain 2-CP. ${ }^{38}$ The lower dose, $10 \mathrm{mg}$, should be considered the no-effect dose for spinal anesthesia, though it provided some transient motor weakness. Similarly, the $20 \mathrm{mg}$ dose did not reliably produce dense motor block, even though it was able to produce a cephalad level of sensory anesthesia of at least L1 in all subjects. ${ }^{38}$

Casati et al tested three different doses (30, 40, and $50 \mathrm{mg}$ ) for intrathecal administration in 45 patients undergoing elective lower limb procedures lasting less than 60 minutes and with a required dermatomeric level at T10. ${ }^{39}$ As expected, spinal block resolution and time to recovery of ambulation results were dose-related. Casati et al included 
Table I Main results of randomized controlled trials published about chloroprocaine between 2004 and 20 I5

\begin{tabular}{|c|c|c|c|c|c|c|}
\hline \multirow{2}{*}{$\begin{array}{l}\text { Author, } \\
\text { year of } \\
\text { publication }\end{array}$} & \multirow[t]{2}{*}{ Setting } & \multirow[t]{2}{*}{ Drug } & \multicolumn{4}{|l|}{ Sensory block } \\
\hline & & & Peak height & $\begin{array}{l}\text { Tourniquet } \\
\text { tolerance }\end{array}$ & $\begin{array}{l}\text { Time until } \\
\text { 2-dermatome } \\
\text { regression }\end{array}$ & $\begin{array}{l}\text { Resolution } \\
\text { of sensory } \\
\text { block }\end{array}$ \\
\hline $\begin{array}{l}\text { Kouri and } \\
\text { Kopacz, } \\
2004^{27}\end{array}$ & $\begin{array}{l}8 \text { healthy volunteers } \\
\text { Age } 35 \pm 6 \text { years } \\
\text { (clinical endpoints } \\
\text { and simulated } \\
\text { discharge pathway) }\end{array}$ & $\begin{array}{l}40 \mathrm{mg} \text { plain chloroprocaine } \\
\text { vs } 40 \mathrm{mg} 2 \% \text { lidocaine }\end{array}$ & $\begin{array}{l}\text { T8 (T5-II) } \\
\text { vs T8 (T6-I2) }\end{array}$ & $\begin{array}{l}46 \pm 6 \mathrm{~min} \\
\text { vs } 38 \pm 24 \mathrm{~min}\end{array}$ & $\begin{array}{l}57 \pm 14 \mathrm{~min} * \\
\text { vs } 73 \pm 23 \mathrm{~min}\end{array}$ & $\begin{array}{l}103 \pm 13 \mathrm{~min}^{*} \\
126 \pm 16 \mathrm{~min} \\
\text { (at S2) }\end{array}$ \\
\hline $\begin{array}{l}\text { Smith et al, } \\
2004^{23}\end{array}$ & $\begin{array}{l}\text { I } 8 \text { healthy } \\
\text { volunteers } \\
\text { Age } 35 \pm 9 \text { years } \\
\text { (clinical endpoints }{ }^{\vee} \\
\text { and simulated } \\
\text { discharge pathway) }\end{array}$ & $\begin{array}{l}30,45 \text {, and } 60 \mathrm{mg} \\
\text { hyperbaric } \\
\text { chloroprocaine } \\
\pm \text { epinephrine }\end{array}$ & $\begin{array}{l}\text { T8 (TIO-T6), } \\
\text { T4 (TII-C6), } \\
\text { TI (T8-C5)* } \\
\text { T7 (L3-T2), } \\
\text { T5 (TIO-TI), } \\
\text { T2 (T6-C5) } \\
(\text { T6-C5) }\end{array}$ & $\begin{array}{l}40 \pm 15 \mathrm{~min} \\
72 \pm 21 \mathrm{~min} \\
71 \pm 12 \mathrm{~min}^{*} \\
37 \pm 11 \mathrm{~min} \\
42 \pm 11 \mathrm{~min} \\
62 \pm 10 \mathrm{~min}^{\mathrm{a}}\end{array}$ & $\begin{array}{l}47 \pm 3 \mathrm{~min} \\
52 \pm 17 \mathrm{~min} \\
57 \pm 3 \mathrm{~min} \\
47 \pm 8 \mathrm{~min} \\
45 \pm 3 \mathrm{~min} \\
43 \pm 5 \mathrm{~min}^{\mathrm{a}}\end{array}$ & $\begin{array}{l}153 \pm 25 \mathrm{~min} \\
162 \pm 33 \mathrm{~min} \\
148 \pm 29 \mathrm{~min}^{*} \\
98 \pm 20 \mathrm{~min} \\
116 \pm 15 \mathrm{~min} \\
132 \pm 23 \mathrm{~min}^{\mathrm{a}}\end{array}$ \\
\hline $\begin{array}{l}\text { Vath and } \\
\text { Kopacz, } \\
2004^{24}\end{array}$ & $\begin{array}{l}8 \text { healthy volunteers } \\
\text { Age } 37 \pm 13 \text { years } \\
\text { (clinical endpoints } \\
\text { and simulated } \\
\text { discharge pathway) }\end{array}$ & $\begin{array}{l}40 \mathrm{mg} \text { chloroprocaine } \\
\pm 20 \mu \mathrm{g} \text { fentanyl }\end{array}$ & $\begin{array}{l}\text { T5 }(\mathrm{T} 7-\mathrm{T} 3)^{*} \\
\text { vs T8 (LI-T4) }\end{array}$ & $\begin{array}{l}5 I \pm 8 \mathrm{~min}^{*} \\
\text { vs } 34 \pm 14 \mathrm{~min}\end{array}$ & $\begin{array}{l}48 \pm 8 \text { min } \\
\text { vs } 45 \pm 16 \text { min }\end{array}$ & $\begin{array}{l}104 \pm 7 \mathrm{~min}^{*} \\
\text { vs } 95 \pm 9 \mathrm{~min}\end{array}$ \\
\hline $\begin{array}{l}\text { Warren } \\
\text { and Kopacz, } \\
2004^{25}\end{array}$ & $\begin{array}{l}8 \text { healthy volunteers } \\
\text { Age } 2 I-48 \text { years } \\
\text { (clinical endpoints } \\
\text { and simulated } \\
\text { discharge pathway) }\end{array}$ & $\begin{array}{l}40 \mathrm{mg} \text { chloroprocaine } \\
\text { with } 0.25 \mathrm{~mL} 10 \% \\
\text { dextrose or } \\
0.25 \mathrm{~mL} \text { saline }\end{array}$ & $\begin{array}{l}\text { T3 (T7-C6) } \\
\text { vs T4 (T7-C7) }\end{array}$ & $\begin{array}{l}45 \pm 7 \mathrm{~min} \\
\text { vs } 42 \pm \mathrm{I} I \mathrm{~min}\end{array}$ & $\begin{array}{l}40 \pm 10 \mathrm{~min} \\
\text { vs } 47 \pm 8 \mathrm{~min}\end{array}$ & $\begin{array}{l}95 \pm 8 \text { min } \\
\text { vs } 96 \pm 9 \text { min }\end{array}$ \\
\hline $\begin{array}{l}\text { Kopacz, } \\
2005^{38}\end{array}$ & $\begin{array}{l}8 \text { healthy volunteers } \\
\text { Age } 40 \pm I \text { I years } \\
\text { (clinical } \\
\text { endpoints }{ }^{\vee} \text { and } \\
\text { simulated discharge } \\
\text { pathway) }\end{array}$ & $\begin{array}{l}10 \mathrm{mg} \text { vs } 20 \mathrm{mg} \\
\text { chloroprocaine }\end{array}$ & $\begin{array}{l}\text { LI (T8-L4) } \\
\text { vs T9 (T4-LI) }\end{array}$ & $\begin{array}{l}7 \pm 6 \mathrm{~min} \\
\text { vs } 22 \pm 9 \text { min }\end{array}$ & $\begin{array}{l}40 \pm 18 \mathrm{~min} \\
\text { vs } 37 \pm 12 \mathrm{~min}\end{array}$ & $\begin{array}{l}49 \pm 10 \mathrm{~min}^{*} \\
\text { vs } 72 \pm 11 \mathrm{~min}\end{array}$ \\
\hline $\begin{array}{l}\text { Davis and } \\
\text { Kopacz, } \\
2005^{26}\end{array}$ & $\begin{array}{l}8 \text { healthy volunteers } \\
\text { Age } 27-60 \text { years } \\
\text { (clinical endpoints } \\
\text { and simulated } \\
\text { discharge pathway) }\end{array}$ & $\begin{array}{l}30 \mathrm{mg} \text { chloroprocaine } \\
\pm 15 \mu \mathrm{g} \text { clonidine }\end{array}$ & $\begin{array}{l}\text { T8 (T4-TII) } \\
\text { vs T8 (T6-L2) }\end{array}$ & $\begin{array}{l}46 \pm 11 \mathrm{~min} \\
\text { vs } 33 \pm 12 \mathrm{~min}\end{array}$ & $\begin{array}{l}50 \pm 9 \text { min } \\
\text { vs } 50 \pm 22 \mathrm{~min}\end{array}$ & $\begin{array}{l}131 \pm 15 \mathrm{~min}^{*} \\
\text { vs } 99 \pm 18 \mathrm{~min}\end{array}$ \\
\hline $\begin{array}{l}\text { Yoos and } \\
\text { Kopacz, } \\
2005^{29}\end{array}$ & $\begin{array}{l}\text { I22 patients } \\
\text { Age } 55 \pm 16 \text { years } \\
\text { Variety of surgical } \\
\text { procedures }\end{array}$ & $\begin{array}{l}\text { Chloroprocaine } \\
20-60 \mathrm{mg} \\
\pm 10-20 \mu \mathrm{g} \text { fentanyl } \\
\text { (rarely dextrose or } \\
\text { water) }\end{array}$ & $\begin{array}{l}\text { Peak T6-T8. } \\
\text { More than TI0 } \\
\text { in all patients }\end{array}$ & NA & NA & NA \\
\hline $\begin{array}{l}\text { Yoos and } \\
\text { Kopacz, } \\
2005^{29}\end{array}$ & $\begin{array}{l}8 \text { healthy volunteers } \\
\text { Age } 38 \pm 7 \text { years } \\
\text { (clinical endpoints } \\
\text { and simulated } \\
\text { discharge pathway) }\end{array}$ & $\begin{array}{l}\text { Chloroprocaine } 40 \mathrm{mg} \\
\text { vs bupivacaine } 7.5 \mathrm{mg}\end{array}$ & $\begin{array}{l}\text { T7 (T3-TI0) } \\
\text { vs T9 (T4-LI) }\end{array}$ & NA & $\begin{array}{l}45 \pm 20 \mathrm{~min} * \\
\text { vs } 74 \pm 20 \mathrm{~min}\end{array}$ & $\begin{array}{l}113 \pm 14 \mathrm{~min}^{*} \\
\text { vs } 191 \pm 30 \mathrm{~min}\end{array}$ \\
\hline $\begin{array}{l}\text { Gonter and } \\
\text { Kopacz, } \\
2005^{28}\end{array}$ & $\begin{array}{l}\text { Healthy volunteers } \\
\text { Age } 42 \pm \text { I I years } \\
\text { (clinical endpoints } \\
\text { and simulated } \\
\text { discharge pathway) }\end{array}$ & $\begin{array}{l}\text { Chloroprocaine } 30 \mathrm{mg} \\
\text { vs procaine } 80 \mathrm{mg}\end{array}$ & $\begin{array}{l}\text { T9 (T6-TI2) } \\
\text { vs T6 (T4-T8) }\end{array}$ & $\begin{array}{l}37 \pm 16 \mathrm{~min} \\
\text { vs } 49 \pm 17 \mathrm{~min}\end{array}$ & $\begin{array}{l}5 I \pm I 7 \mathrm{~min} \\
\text { vs } 53 \pm 10 \mathrm{~min}\end{array}$ & $\begin{array}{l}103 \pm 12 \mathrm{~min} * \\
\text { vs } 151 \pm 26 \mathrm{~min}\end{array}$ \\
\hline $\begin{array}{l}\text { Casati et al, } \\
2006^{39}\end{array}$ & $\begin{array}{l}45 \text { ASA I-II outpatients } \\
\text { Age } 59 \pm 13,56 \pm 14 \\
48 \pm 16 \text { years } \\
\text { Lower limb surgery }\end{array}$ & $\begin{array}{l}\text { Chloroprocaine } 30,40 \text {, } \\
\text { and } 50 \mathrm{mg}\end{array}$ & NA & NA & NA & $\begin{array}{l}60(4 \mathrm{I}-98) \mathrm{min}, * \\
85(46-141) \mathrm{min}, \\
97(60-169) \mathrm{min}^{\mathrm{b}}\end{array}$ \\
\hline
\end{tabular}




\begin{tabular}{|c|c|c|c|c|}
\hline \multicolumn{2}{|l|}{ Motor block } & \multicolumn{2}{|c|}{ Discharge time } & \multirow{2}{*}{$\begin{array}{l}\text { Complications } \\
\text { Complications }\end{array}$} \\
\hline $\begin{array}{l}\text { Time until } \\
\text { recovery } \\
\text { at abdomen }\end{array}$ & $\begin{array}{l}\text { Time until } \\
\text { recovery - Bromage } \\
\text { Scale }\end{array}$ & Ambulation & Micturition & \\
\hline $\begin{array}{l}71 \pm 18 \mathrm{~min} \\
\text { vs } 60 \pm 53 \mathrm{~min}\end{array}$ & $\begin{array}{l}79 \pm 15 \mathrm{~min} \\
\text { vs } 90 \pm 14 \mathrm{~min}\end{array}$ & $\begin{array}{l}104 \pm 12 \text { min* } \\
\text { vs } 134 \pm 14 \text { min }\end{array}$ & $\begin{array}{l}104 \pm 12 \mathrm{~min} * \\
\text { vs } 134 \pm 14 \mathrm{~min}\end{array}$ & $\begin{array}{l}\text { TNS in } 7 \text { out of } 8 \text { patients with lidocaine } \\
\text { No TNS with chloroprocaine }\end{array}$ \\
\hline
\end{tabular}

\begin{tabular}{|c|c|c|c|c|}
\hline $60 \pm 65 \mathrm{~min}$ & $90 \pm 30 \mathrm{~min}$ & $158 \pm 33 \mathrm{~min}$ & $167 \pm 47 \mathrm{~min}$ & Nonspecific flu-like symptoms, radiating back pain (2 \\
\hline $88 \pm 43 \mathrm{~min}$ & $|3| \pm 46 \mathrm{~min}$ & $162 \pm 33 \mathrm{~min}$ & $161 \pm 33 \mathrm{~min}$ & $\mathrm{pt})$, non-radiating low back ache (4 pt) after $2-\mathrm{CP}$ and \\
\hline $80 \pm 26$ min* & $120 \pm 30 \mathrm{~min}^{*}$ & $151 \pm 33$ min* & $164 \pm 24$ min* & epinephrine non-radiating low back pain (I pt) after \\
\hline $43 \pm 26 \mathrm{~min}$ & $72 \pm 12 \mathrm{~min}$ & $100 \pm 20 \mathrm{~min}$ & $100 \pm 21 \mathrm{~min}$ & $2-C P$ \\
\hline $56 \pm 9 \mathrm{~min}$ & $88 \pm 15 \mathrm{~min}$ & $119 \pm 15 \mathrm{~min}$ & $132 \pm 19 \mathrm{~min}$ & \\
\hline $78 \pm 16 \min ^{\mathrm{a}}$ & $100 \pm 13 \mathrm{~min}^{\mathrm{a}}$ & $133 \pm 20 \mathrm{~min}^{\mathrm{a}}$ & $|4| \pm 2 \mid \mathrm{min}^{\mathrm{a}}$ & \\
\hline $6 I \pm I I \min$ & $81 \pm 16$ min* & $104 \pm 7 \mathrm{~min}^{*}$ & $104 \pm 7$ min* & Itching (no treatment required) when fentanyl was used \\
\hline vs $49 \pm 10 \mathrm{~min}$ & vs $67 \pm 13 \mathrm{~min}$ & vs $95 \pm 9 \mathrm{~min}$ & vs $95 \pm 9 \mathrm{~min}$ & \\
\hline
\end{tabular}

\begin{tabular}{|c|c|c|c|c|}
\hline $\begin{array}{l}64 \pm 12 \text { min } \\
\text { vs } 60 \pm 19 \text { min }\end{array}$ & $\begin{array}{l}80 \pm 14 \text { min } \\
\text { vs } 81 \pm 15 \text { min }\end{array}$ & $\begin{array}{l}96 \pm 7 \text { min } \\
\text { vs } 96 \pm 9 \text { min }\end{array}$ & $\begin{array}{l}101 \pm 7 \text { min } \\
\text { vs } 96 \pm 9 \text { min }\end{array}$ & $\begin{array}{l}\text { Greater residual bladder volumes in the dextrose } \\
\text { group }\end{array}$ \\
\hline
\end{tabular}

NA

$16 \pm 15 \mathrm{~min}^{*}$

$44 \pm 19 \mathrm{~min}^{*}$

$50 \pm 10 \min ^{*}$

No TNS

vs $48 \pm 7$ min

vs $73 \pm 9 \mathrm{~min}$

vs $73 \pm 9 \mathrm{~min}$

NA

$79 \pm 19 \mathrm{~min}^{*}$

vs $65 \pm 13 \mathrm{~min}$

$131 \pm 15$ min*

vs $99 \pm 18 \mathrm{~min}$

$|3| \pm 15 \mathrm{~min}^{*}$

vs $99 \pm 18 \mathrm{~min}$

NA

NA

$155 \pm 35 \mathrm{~min}$

$207.9 \pm 69.4 \mathrm{~min}$

(discharge)

NA

$81 \pm 15 \mathrm{~min}^{*}$
vs $138 \pm 24 \mathrm{~min}$

$113 \pm 14 \mathrm{~min}^{*}$

vs $191 \pm 30 \mathrm{~min}$

$113 \pm 14 \mathrm{~min}^{*}$

vs $191 \pm 32 \mathrm{~min}$

NA

$$
54 \pm 23 \mathrm{~min}
$$

vs $55 \pm 44 \mathrm{~min}$

$103 \pm 12 \mathrm{~min}^{*}$

vs $|5| \pm 26 \mathrm{~min}$

$103 \pm 12 \mathrm{~min}^{*}$

vs $156 \pm 23 \mathrm{~min}$

NA

NA

85 (45-198) min,*

$180(72-281) \mathrm{min}$,

$185(90-355) \mathrm{min}^{\mathrm{b}}$
$182(120-267) \mathrm{min}$,
$198(|23-27|) \mathrm{min}$,
$203(102-394) \mathrm{min}^{\mathrm{b}}$
II patients complained of nausea in the PACU No TNS

No TNS

Pruritus in I patient and dysphoria in I patient (both in the procaine group)

No TNS 
Table I (Continued)

\begin{tabular}{|c|c|c|c|c|c|c|}
\hline \multirow{2}{*}{$\begin{array}{l}\text { Author, } \\
\text { year of } \\
\text { publication }\end{array}$} & \multirow[t]{2}{*}{ Setting } & \multirow[t]{2}{*}{ Drug } & \multicolumn{4}{|l|}{ Sensory block } \\
\hline & & & Peak height & $\begin{array}{l}\text { Tourniquet } \\
\text { tolerance }\end{array}$ & $\begin{array}{l}\text { Time until } \\
\text { 2-dermatome } \\
\text { regression }\end{array}$ & $\begin{array}{l}\text { Resolution } \\
\text { of sensory } \\
\text { block }\end{array}$ \\
\hline $\begin{array}{l}\text { Casati et al, } \\
2007^{40}\end{array}$ & $\begin{array}{l}30 \text { ASA patients } \\
\text { Age I8-70 years } \\
\text { Knee arthroscopy }\end{array}$ & $\begin{array}{l}\text { Chloroprocaine } 50 \mathrm{mg} \\
\text { vs lidocaine } 50 \mathrm{mg}\end{array}$ & NA & NA & NA & $\begin{array}{l}\text { I } 20(80-\mid 75) \mathrm{min}^{*} \\
\text { vs } 95(68-\mid 70) \mathrm{min}\end{array}$ \\
\hline $\begin{array}{l}\text { Sell et al, } \\
2008^{37}\end{array}$ & $\begin{array}{l}64 \text { ASA I-III patients } \\
\text { Age I8-80 years } \\
\text { Lower limb surgery }\end{array}$ & $\begin{array}{l}\text { Chloroprocaine } 35,40,45 \text {, } \\
\text { and } 50 \mathrm{mg}\end{array}$ & T9 in all groups & NA & NA & $\begin{array}{l}\text { III }(96-126) \mathrm{min}, \\
\text { I08 (95-12I) min,* } \\
\text { I } 28(116-138) \mathrm{min}, \\
\text { I34 (I23-145) minc }\end{array}$ \\
\hline $\begin{array}{l}\text { Lacasse et al, } \\
20 \mathrm{II}^{44}\end{array}$ & $\begin{array}{l}\text { I06 ASA I-III patients } \\
\text { Age }>18 \text { years } \\
\text { Short ambulatory } \\
\text { procedures }\end{array}$ & $\begin{array}{l}\text { Chloroprocaine } 40 \mathrm{mg} \\
\text { vs bupivacaine } 7.5 \mathrm{mg}\end{array}$ & $\begin{array}{l}\text { T7 (TI-TI0) } \\
\text { vs T7 (TI-TII) }\end{array}$ & NA & $\begin{array}{l}50 \pm 18 \mathrm{~min}^{*} \\
\text { vs } 75 \pm 37 \mathrm{~min}\end{array}$ & $\begin{array}{l}146 \pm 38 \mathrm{~min}^{*} \\
\text { vs } 329 \pm 82 \mathrm{~min}\end{array}$ \\
\hline $\begin{array}{l}\text { Forster et al, } \\
20 \mathrm{II}^{13}\end{array}$ & $\begin{array}{l}70 \text { ASA I-II patients } \\
\text { Age } \leq 65 \text { years } \\
\text { Day-case knee } \\
\text { arthroscopy }\end{array}$ & $\begin{array}{l}\text { Chloroprocaine } 40 \mathrm{mg} \\
\text { vs articaine } 60 \mathrm{mg}\end{array}$ & $\begin{array}{l}\text { TIO (TI2-T6) } \\
\text { vs TIO (TII-T5) }\end{array}$ & NA & $\begin{array}{l}60(45-75) \mathrm{min} \text { vs } \\
60(45-75) \mathrm{min}\end{array}$ & $\begin{array}{l}105(105-135) \mathrm{min}^{*} \\
\text { vs } \\
165(135-180) \mathrm{min}\end{array}$ \\
\hline $\begin{array}{l}\text { Vaghadia et al, } \\
2012^{4 !}\end{array}$ & $\begin{array}{l}40 \text { ASA I-III patients } \\
\text { Age 59-78 years } \\
\text { TURP }\end{array}$ & $\begin{array}{l}\text { Chloroprocaine } 40 \mathrm{mg}+ \\
\text { I2.5 } \mathrm{gg} \text { fentanyl } \\
\text { vs } 35 \mathrm{mg} \text { lidocaine }+ \\
12.5 \mu \mathrm{g} \text { fentanyl }\end{array}$ & $\begin{array}{l}\text { T8 in both } \\
\text { groups }\end{array}$ & NA & NA & $\begin{array}{l}155 \pm 55 \text { min vs } \\
163 \pm 37 \text { min }\end{array}$ \\
\hline $\begin{array}{l}\text { Forster et al, } \\
2013^{43}\end{array}$ & $\begin{array}{l}36 \text { ASA I-III patients } \\
\text { Age I8-70 years } \\
\text { Day-case knee } \\
\text { surgery }\end{array}$ & $\begin{array}{l}\text { Chloroprocaine } 40 \mathrm{mg} \\
\text { vs articaine } 40 \mathrm{mg}\end{array}$ & $\begin{array}{l}\text { T8 (TI0-T7) vs } \\
\text { T8 (TI2-T6) }\end{array}$ & NA & $\begin{array}{l}60(45-60) \mathrm{min}^{*} \\
\text { vs } \\
75(49-75) \mathrm{min}\end{array}$ & $\begin{array}{l}\text { I05 }(90-124) \mathrm{min}^{*} \\
\text { vs } \\
\text { I35 (109-176) min }\end{array}$ \\
\hline $\begin{array}{l}\text { Breebart et al, } \\
2014^{42}\end{array}$ & $\begin{array}{l}\text { I00 ASA I-II patients } \\
\text { Age, mean (SD): } \\
44 \text { (I2); } 46 \text { (I4); } \\
43 \text { (I2); } 5 \text { I (I2) years } \\
\text { Knee arthroscopy }\end{array}$ & $\begin{array}{l}\text { Lidocaine } 60 \mathrm{mg} \pm 500 \mathrm{~mL} \\
\text { iv preload (lidocaine+; } \\
\text { lidocaine-)Chloroprocaine } \\
40 \mathrm{mg} \pm 500 \mathrm{~mL} \text { iv preload } \\
(\mathrm{CP}+\text {; } \mathrm{CP}-\text { ) }\end{array}$ & $\begin{array}{l}\mathrm{T} 5 \\
\mathrm{~T} 5 \\
\mathrm{~T} 7 \\
\mathrm{~T} 7^{\mathrm{d}}\end{array}$ & NA & NA & $\begin{array}{l}140(46) \mathrm{min}^{\#} \\
129(37) \mathrm{min}^{\prime \#} \\
92(25) \mathrm{min}_{,, *} \\
86(21) \mathrm{min}, \#, *, \mathrm{~d}\end{array}$ \\
\hline $\begin{array}{l}\text { Camponovo } \\
\text { et al, } 2014^{45}\end{array}$ & $\begin{array}{l}\text { I } 30 \text { ASA I-II patients } \\
\text { Age I8-80 years } \\
\text { Lower abdominal or } \\
\text { lower limb procedures } \\
\text { (TI } 0 \text { level required) }\end{array}$ & $\begin{array}{l}\text { Chloroprocaine } 50 \mathrm{mg} \\
\text { vs bupivacaine } 10 \mathrm{mg}\end{array}$ & $\begin{array}{l}\text { TI0 (TI0-T3) vs } \\
\text { TIO (TI0-T2) }\end{array}$ & NA & NA & $\begin{array}{l}105(60-194) \mathrm{min}^{*} \\
\text { vs } \\
225(130-442) \mathrm{min}\end{array}$ \\
\hline
\end{tabular}

Notes: Data are shown as mean \pm standard deviation or median ( 25 th-75th percentiles), unless stated otherwise. *Significant versus other treatment/group. \#Regression to L2. "Gastrocnemius dynamometry ( $90 \%$ of baseline) (minutes). ${ }^{\wedge}$ Time to hospital discharge. ${ }^{a}$ The first three rows of data are results for hyperbaric chloroprocaine 30 , 45 and $60 \mathrm{mg}$ with epinephrine, and the next three rows of data are results for 30,45 and $60 \mathrm{mg}$ without epinephrine. ${ }^{\mathrm{b}}$ The three rows or items of data are the results for chloroprocaine 30,40 , and $50 \mathrm{mg}$, respectively. ${ }^{\mathrm{C}}$ The four rows of data are the results for chloroprocaine $35,40,45$, and $50 \mathrm{mg}$, respectively. ${ }^{\mathrm{D}} \mathrm{The}$ four rows or items of data are the results for lidocaine+, lidocaine-, $\mathrm{CP}+$, and $\mathrm{CP}-$, respectively.

Abbreviations: 2-CP, 2-chloroprocaine; ASA, American Society of Anesthesiologists; $\mathrm{CP}$, chloroprocaine; $\mathrm{CP}+$, chloroprocaine with iv preload; $\mathrm{CP}-$, chloroprocaine without iv preload; iv, intravenous; min, minutes; lidocaine+, lidocaine with iv preload; lidocaine-, lidocaine without iv preload; NA, not available; PACU, post-anesthesia care unit; PDPH, post-dural puncture headache; POD, postoperative day; PONV, postoperative nausea and vomiting; pt, patient(s); TNS, transient neurological symptoms; TURP, transurethral resection of the prostate.

patients undergoing procedures lasting between 45 and 60 minutes, finding that $33 \%$ of patients in the $30 \mathrm{mg}$ group required intraoperative analgesic supplementation as a result of insufficient analgesia. The authors concluded that the $30 \mathrm{mg}$ dose may not be suitable for lower limb procedures lasting $\leq 60$ minutes. ${ }^{39}$
Yoos and Kopacz found instead an increasing tendency toward the administration of the lowest dose of $30 \mathrm{mg}$ of 2-CP in their ambulatory setting, and a decrease in the addition of fentanyl among their anesthesiologists, indicating that the correct patient and surgery selections allow a successful use also of the $30 \mathrm{mg}$ dose..$^{29}$ 


\begin{tabular}{|c|c|c|c|c|}
\hline \multicolumn{2}{|l|}{ Motor block } & \multicolumn{2}{|l|}{ Discharge time } & \multirow{2}{*}{$\begin{array}{l}\text { Complications } \\
\text { Complications }\end{array}$} \\
\hline $\begin{array}{l}\text { Time until } \\
\text { recovery } \\
\text { at abdomen }\end{array}$ & $\begin{array}{l}\text { Time until } \\
\text { recovery - Bromage } \\
\text { Scale }\end{array}$ & Ambulation & Micturition & \\
\hline NA & $\begin{array}{l}100(60-140) \mathrm{min}^{*} \\
\text { vs } 60(45-120) \mathrm{min}\end{array}$ & $\begin{array}{l}152(100-185) \mathrm{min}^{*} \\
\text { vs } 103(70-191) \mathrm{min}\end{array}$ & $\begin{array}{l}190(148-340) \min \\
\text { vs } 180(100-354) \min \end{array}$ & $\begin{array}{l}\text { TNS in } 33 \% \text { of patients in the lidocaine group. } \\
\text { No TNS with chloroprocaine }\end{array}$ \\
\hline NA & $\begin{array}{l}106(91-121) \mathrm{min} \\
100(99-123) \mathrm{min} \\
111(99-123) \mathrm{min} \\
119(102-137) \mathrm{min}^{\mathrm{c}}\end{array}$ & $\begin{array}{l}117(103-131) \mathrm{min}, * \\
116(103-130) \mathrm{min}, \\
127(106-148) \mathrm{min} \\
144(128-161) \mathrm{min}^{\mathrm{c}}\end{array}$ & $\begin{array}{l}123(108-138) \mathrm{min}, * \\
122(109-135) \mathrm{min}, \\
137(124-149) \mathrm{min} \\
165(141-189) \mathrm{min}^{\mathrm{c}}\end{array}$ & No TNS \\
\hline NA & $\begin{array}{l}76 \pm 25 \mathrm{~min}^{*} \\
\text { vs } 119 \pm 93 \mathrm{~min}\end{array}$ & $\begin{array}{l}225 \pm 56 \mathrm{~min}^{*} \\
\text { vs } 265 \pm 65 \mathrm{~min}\end{array}$ & $\begin{array}{l}27 I \pm 96 \mathrm{~min}^{*} \\
\text { vs } 338 \pm 99 \mathrm{~min}\end{array}$ & $\begin{array}{l}\text { I post-dural puncture headache per group, I TNS per } \\
\text { group, } 45 \% \text { back ache after } 2 \text {-CP and } 38 \% \text { after } \\
\text { bupivacaine }\end{array}$ \\
\hline NA & $\begin{array}{l}75(60 / 90) \mathrm{min}^{*} \\
\text { vs I35 (105/I50) min }\end{array}$ & $\begin{array}{l}318 \pm 74.2 \mathrm{~min} \\
\text { vs } 392 \pm 93.2 \mathrm{~min}^{\wedge}\end{array}$ & $\begin{array}{l}204 \pm 61.8 \mathrm{~min} \\
\text { vs } 219 \pm 71.6 \mathrm{~min}\end{array}$ & No TNS \\
\hline $8 I \pm 4 I \min$ & $117 \pm 36 \min$ & NA & NA & 4 patients developed TNS with lidocaine \\
\hline $7 I \pm 4 I \min$ & $120 \pm 35 \mathrm{~min}$ & & & $\begin{array}{l}\text { I patient developed cauda equina syndrome after } \\
\text { chloroprocaine fully recovered after some weeks }\end{array}$ \\
\hline NA & $\begin{array}{l}75(7 / / 90) \min ^{*} \\
\text { vs } 105(94 / / 20) \min \end{array}$ & NA & $\begin{array}{l}\text { I7I }(163 / 197) \min \\
\text { vs } 236(152 / 279) \min \end{array}$ & $\begin{array}{l}5 \text { patients had PONV in both groups on first POD } \\
\text { I patient had PONV after articaine vs } \\
4 \text { patients after chloroprocaine on POD } 7 \\
2 \text { patients after articaine vs } 3 \text { after chloroprocaine had } \\
\text { non-PDPH on POD I } \\
3 \text { patients after articaine vs I patient after } \\
\text { chloroprocaine had non-PDPH on POD } 7 \\
\text { I patient showed TNS after articaine on POD I }\end{array}$ \\
\hline NA & 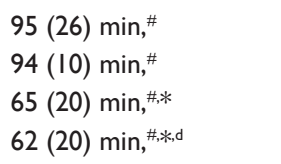 & NA & $\begin{array}{l}210(55) \mathrm{min} \\
205(58) \mathrm{min} \\
161(36) \mathrm{min}, \\
175(5 \mathrm{I}) \mathrm{min}^{\mathrm{d}}\end{array}$ & I patient complained of TNS after lidocaine \\
\hline NA & $\begin{array}{l}100(40-194) \mathrm{min}^{*} \text { vs } \\
210(120-415) \mathrm{min}\end{array}$ & $\begin{array}{l}\text { I } 42.5(86-454) \mathrm{min}^{*} \mathrm{vs} \\
290.5(190-490) \mathrm{min}\end{array}$ & NA & $\begin{array}{l}\text { I patient experienced foot cramps after bupivacaine } \\
\text { I patient reported non-PDPH after bupivacaine } \\
\text { I patient reported injection site pain after bupivacaine } \\
\text { No TNS was reported }\end{array}$ \\
\hline
\end{tabular}

\section{2-CP versus other fast-acting local anesthetics}

In the retrospective review by Hejtmanek and Pollock, ${ }^{30} 2-\mathrm{CP}$ showed a faster profile than lidocaine in terms of time from injection to ambulation and time from injection to hospital discharge with a comparable incidence of urinary retention.
The comparison with lidocaine, as well as with other short-acting local anesthetics, has been extensively evaluated in literature. Kouri and Kopacz compared intrathecal injection of $40 \mathrm{mg} 2 \%$ lidocaine with $40 \mathrm{mg} 2 \%$ 2-CP in eight healthy volunteers and demonstrated a faster profile for 2-CP, demonstrating shorter resolution time of sensory block and 
a significantly shorter time to complete block regression and voiding. ${ }^{27}$ Consistently with these volunteer data, Casati et al found a faster recovery profile of sensory and motor blocks and faster time-to-ambulation after 2-CP than after an equal dose of lidocaine in 30 patients undergoing knee arthroscopy. No significant differences were noted in times for hospital discharge due to comparable times to spontaneous voiding, which was required as a criterion for patients' discharge. ${ }^{40}$

Also Vaghadia et al compared lidocaine and 2-CP in combination with fentanyl, to provide selective spinal anesthesia for outpatient transurethral resection of the prostate. The authors did not find any statistical difference between the two groups with respect to onset and offset of spinal block variables. The authors reported four cases of transient neurological symptoms after lidocaine and one case of cauda andom-like syndrome after CP in a 66-year old patient, who developed symptoms within 24 hours after an uneventful L3-L4 spinal anesthesia with a $25 \mathrm{G}$ needle, persisting for several weeks. The patient complained of numbness in both buttocks extending down the posterior thighs to both feet, thigh weakness, stabbing pain in his anterior legs bilaterally requiring treatment with oral oxycodone, and urinary retention. Nevertheless, the patient recovered after some weeks. ${ }^{41}$

A more recent study by Breebart et al, randomized 100 patients undergoing day-case arthroscopy to receive either lidocaine $60 \mathrm{mg}$ or CP $40 \mathrm{mg}$ intrathecally with or without a $500 \mathrm{~mL}$ pre-load of crystalloid. The authors found no differences in voiding time within the $\mathrm{CP}$ or lidocaine groups, although discharge and micturition was faster with CP than lidocaine. When looking at subgroups, only the CP group receiving pre-load was discharged faster than both the lidocaine groups and more serious micturition problems (requiring single in and out bladder evacuation) occurred in the lidocaine group with pre-load compared with both CP groups, demonstrating a more favorable profile for $\mathrm{CP}$ in the ambulatory setting. ${ }^{42}$

CP $30 \mathrm{mg}$ was also compared to another fast-acting local anesthetic, procaine $80 \mathrm{mg}$, showing similar surgical efficacy but significantly shorter sensory block and discharge times. ${ }^{28}$

Förster et al compared articaine $40 \mathrm{mg}$ to $\mathrm{CP} 40 \mathrm{mg}$ in 36 patients scheduled for day-case knee arthroscopy. The authors found comparable onset and maximal spread of spinal anesthesia, while offset was significantly slower with articaine than $\mathrm{CP}^{43}$

\section{CP versus bupivacaine}

2-CP has been compared to bupivacaine in the studies by Yoos and $\mathrm{Kopacz}^{35}$ and Lacasse et al. ${ }^{44}$ In the first study, 40 $\mathrm{mg}$ of 2-CP was compared with $7.5 \mathrm{mg}$ of bupivacaine in a double-blind, randomized, crossover, volunteer study in terms of pinprick anesthesia, motor strength, tolerance to tourniquet and electrical stimulation, and simulated discharge criteria. ${ }^{37}$ Lacasse et al compared $7.5 \mathrm{mg}$ of hyperbaric bupivacaine $0.75 \%$ to $40 \mathrm{mg}$ of $2-\mathrm{CP} 2 \%$ in 106 patients. ${ }^{44}$ The authors in both studies found significantly longer discharge times with low-dose bupivacaine than with 2-CP. All offset variables showed a faster resolution of the spinal block after 2-CP, including time for two-segment regression, time for regression to $\mathrm{L} 1$, time for complete regression to $\mathrm{S} 2$, duration of motor blockade, time-to-ambulation as well as time to first analgesic requirement. Lacasse et al reported one case of TNS after spinal 2-CP and one case after spinal bupivacaine, both identified at the 24-hour follow-up phone call. Symptoms were defined as pain or dysesthesia in the legs and/or buttocks in the first 24 hours after recovery in two female patients aged 50 to 60 years old who underwent transobturator tension-free urethral suspension in the lithotomy position. As claimed by the authors, differential diagnosis includes the well-described neuropathies known to be associated with the lithotomy position as well as surgical trauma due to entrapment of the obturator nerve during placement of the sling. The authors could therefore not be conclusive about the diagnosis of TNS. ${ }^{44}$ Patients receiving intrathecal 2-CP demonstrated faster times to micturition and times between first try and successful voiding. Camponovo et al also performed a clinical study demonstrating that spinal anesthesia performed with $50 \mathrm{mg}$ of plain $1 \% 2-\mathrm{CP}$ provides adequate spinal anesthesia for lower abdominal and lower limb outpatient procedures lasting less than 40 minutes, with faster recovery from anesthesia and eligibility for home discharge in comparison with $10 \mathrm{mg}$ of plain $0.5 \%$ bupivacaine. ${ }^{45}$ The authors found onset time to be almost the same with 1\% 2-CP $50 \mathrm{mg}$ and $10 \mathrm{mg}$ bupivacaine $0.5 \%$ and confirmed faster offset times after 2-CP spinal anesthesia. ${ }^{45}$

\section{Conclusion}

The availability of reliable and safe short-acting local anesthetics has recently renewed interest in spinal technique for outpatient surgery, offering an alternative to general anesthesia.

Intrathecal $1 \%$ or $2 \% 2-\mathrm{CP}$ represents an interesting alternative to lidocaine for surgical blocks and short or ultra-short surgical procedures. When compared with spinal bupivacaine, it resulted in a significantly faster offset of sensory and motor blocks with similar onset time. The safety of intrathecal use of the preservative-free 2-CP formulation is currently sustained both by volunteer and clinical studies. Literature suggests a dose ranging between 
30 and $60 \mathrm{mg}$ for procedures lasting 60 minutes or less, while $10 \mathrm{mg}$ is considered the no-effect dose. Further investigations are necessary to address the adequacy of the lower recommended dose of $30 \mathrm{mg}$ in ultra-short procedures lasting 40 minutes of the lower extremities (required dermatome level at T12).

\section{Disclosure}

Stefano Bonarelli and Daniela Ghisi report no conflicts of interest in this work.

\section{References}

1. Dunning K, Liedtke E, Toedter L, Rohatgi C. Outpatient surgery centers draw cases away from hospitals, impact resident training volume. J Surg Educ. 2008;65(6):460-644.

2. Apfelbaum JL, Walawander CA, Grasela TH, et al. Eliminating intensive postoperative care in same-day surgery patients using short-acting anesthetics. Anesthesiology. 2002;97(1):66-74.

3. Förster JG. Short-acting spinal anesthesia in the ambulatory setting. Curr Opin Anaesthesiol. 2014;27(6):597-604.

4. Alley EA, Mulory MF. Neuraxial anesthesia for outpatients. Anesthesiol Clin. 2014;32(2):357-369.

5. Mulroy MF, Salinas FV, Larkin KL, Polissar NL. Ambulatory surgery patients may be discharged before voiding after short-acting spinal and epidural anesthesia. Anesthesiology. 2002;97(2):315-319.

6. Förster JG, Rosenberg PH. Revival of old local anesthetics for spinal anesthesia in ambulatory surgery. Curr Opin Anaesthesiol. 2011; 24(6):633-637.

7. Fanelli A, Ghisi D, Allegri M. Is spinal anaesthesia a suitable technique for ultra-short outpatient procedures? Acta Biomed. 2013; 84(1):76-80.

8. Gupta A, Stierer T, Zuckerman R, Sakima N, Parker SD, Fleisher LA. Comparison of recovery profile after ambulatory anesthesia with propofol, isoflurane, sevoflurane and desflurane: a systematic review. Anesth Analg. 2004;98(3):632-634.

9. Liu SS, Strodtbeck WM, Richman JM, Wu CL. A Comparison of Regional Versus General Anesthesia for Ambulatory Anesthesia: A Meta-Analysis of Randomized Controlled Trials. Anesth Analg. 2005;101(6):1634-1642.

10. Liu SS, Ware PD, Allen HW, Neal JM, Pollock JE. Dose-response characteristics of spinal bupivacaine in volunteers: clinical implications for ambulatory anesthesia. Anesthesiology. 1996;85(4):729-736.

11. Pollock JE. Transient neurologic symptoms: etiology, risk factors, and management. Reg Anesth Pain Med. 2002;27(6):581-586.

12. Freedman JM, Li DK, Drasner K, Jaskela MC, Larsen B, Wi S. Transient neurologic symptoms after spinal anesthesia: an epidemiologic study of 1863 patients. Anesthesiology. 1998;89(6):633-641.

13. Förster JG, Kallio H, Rosenberg PH, HarilainenA, Sandelin J, Pitkänen MT. Chloroprocaine vs articaine as spinal anaesthetics for day-case knee arthroscopy. Acta Anaesthesiol Scand. 2011;55(3):273-281.

14. Foldes FF, McNall PG. 2-Chloroprocaine: a new local anesthetic agent. Anesthesiology.1952;13(3):287-296.

15. Winnie AP, Nader AM. Santayana's prophecy fulfilled. Reg Anesth Pain Med. 2001;26(6):558-564.

16. Ravindran RS, Bond VK, Tasch MD, Gupta CD, Luerssen TG. Prolonged neural blockade following regional anesthesia with 2-chloroprocaine. Anesth Analg. 1980;59(6):447-451.

17. Reisner LS, Hochman BN, Plumer MH. Persistent neurologic deficit and adhesive arachnoiditis following intrathecal 2-chloroprocaine. Anesth Analg. 1980;59(6):452-454.

18. Moore DC, Spierdijk J, vanKleef JD, Coleman RL, Love GF. Chloroprocaine toxicity: four additional cases. Anesth Analg. 1982; 61(2):158-159.
19. Wang BC, Hillman DE, Spielholz NI, Turndorf H. Chronic neurological deficits and Nesacaine-CE - an effect of the anesthetic, 2-chloroprocaine, or the antioxidant, sodium bisulfite? Anesth Analg. 1984;63(4):445-447.

20. Taniguchi M, Bollen AW, Drasner K. Sodium bisulfite: scapegoat for chloroprocaine neurotoxicity? Anesthesiology. 2004;100(1):85-91.

21. Baker MT. Chloroprocaine or sulfite toxicity? Anesthesiology. 2004; 101(5):1247.

22. Cabré F, Marín C, Cascante M, Canela EI. Occurrence and comparison of sulfite oxidase activity in mammalian tissues. Biochem Med Metab Biol. 1990;43(2):159-162.

23. Smith KN, Kopacz DJ, McDonald SB. Spinal 2-chloroprocaine: a doseranging study and the effect of added epinephrine. Anesth Analg. 2004;98(1):81-88

24. Vath JS, Kopacz DJ. Spinal 2-chloroprocaine: the effect of added fentanyl. Anesth Analg. 2004;98(1):89-94.

25. Warren DT, Kopacz DJ. Spinal 2-chloroprocaine: the effect of added dextrose. Anesth Analg. 2004;98:95-101.

26. Davis BR, Kopacz DJ. Spinal 2-chloroprocaine: the effect of added clonidine. Anesth Analg. 2005;100(2):559-565.

27. Kouri M, Kopacz DJ. Spinal 2-chloroprocaine: a comparison with lidocaine in volunteers. Anesth Analg. 2004;98(1):75-80.

28. Gonter AF, Kopacz DJ. Spinal 2-chloroprocaine: a comparison with procaine in volunteers. Anesth Analg. 2005;100(2):573-579.

29. Yoos JR, Kopacz DJ. Spinal 2-chloroprocaine for surgery: an initial 10-month experience. Anesth Analg. 2005;100(2):553-558.

30. Hejtmanek MR, Pollock JE. Chloroprocaine for spinal anesthesia: a retrospective analysis. Acta Anaesth Scand. 2011;55(3):267-272.

31. Pavlin DJ, Pavlin EG, Fitzgibbon DR, Koerschgen ME, Plitt TM. Management of bladder function after outpatient surgery. Anesthesiology. 1999;91(1):42-50.

32. Choi S, Mahon P, Awad IT. Neuraxial anesthesia and bladder dysfunction in the perioperative period: a systematic review. Can J Anaesth. 2012;59(7):681-703.

33. Kamphuis ET, Ionescu TI, Kuipers PW, de Gier J, van Venrooij GE, Boon TA. Recovery of storage and emptying functions of the urinary bladder after spinal anesthesia with lidocaine and with bupivacaine in men. Anesthesiology. 1998;88(2):310-316.

34. Mulroy MF. Outpatients do not need to void after short neuraxial blocks. Anesthesiology. 2009;111(6):1388.

35. Yoos JR, Kopacz DJ. Spinal 2-chloroprocaine: a comparison with smalldose bupivacaine in volunteers. Anesth Analg. 2005;100(2):566-572.

36. Etezadi F, Karimi Yarandi K, Ahangary A, et al. The effect of needle type, duration of surgery and position of the patient on the risk of transient neurologic symptoms. Anesth Pain Med. 2013;2(4):154-158.

37. Sell A, Tein T, Pitkänen M. Spinal 2-chloroprocaine: effective dose for ambulatory surgery. Acta Anaesthesiol Scand. 2008;52(5):695-699.

38. Kopacz DJ. Spinal 2-chloroprocaine: minimum effective dose. Reg Anesth Pain Med. 2005;30(1):36-42.

39. Casati A, Danelli G, Berti M, et al. Intrathecal 2-chloroprocaine for lower limb outpatient surgery: a prospective, randomized, double-blind, clinical evaluation. Anesth Analg. 2006;103(1):234-238.

40. Casati A, Fanelli G, Danelli G, et al. Spinal anesthesia with lidocaine or preservative-free 2-chlorprocaine for outpatient knee arthroscopy: a prospective, randomized, double-blind comparison. Anesth Analg. 2007;104(4):959-964.

41. Vaghadia H, Neilson G, Lennox PH. Selective spinal anesthesia for outpatient transurethral prostatectomy (TURP): randomized controlled comparison of chloroprocaine with lidocaine. Acta Anaesthesiol Scand. 2012;56(2):217-223.

42. Breebaart MB, Teune A, Sermeus LA, Vercauteren MP. Intrathecal chloroprocaine vs lidocaine in day-case surgery: recovery, discharge and effect of prehydration on micturition. Acta Anaesthesiol Scand. 2014;58(2):206-213.

43. Förster JG, Rosenberg PH, Harilainen A, Sandelin A, Pitkänen A. Chloroprocaine $40 \mathrm{mg}$ produces shorter spinal block than articaine $40 \mathrm{mg}$ in day-case knee arthroscopy patients. Acta Anaesthesiol Scand. 2013;57(7):911-919. 
44. Lacasse MA, Roy JD, Forget J, et al. Comparison of bupivacaine and 2-chloroprocaine for spinal anesthesia for outpatient surgery: a double-blind randomized trial. Can J Anesth. 2011;58(4): 384-391.
45. Camponovo C, Wulf H, Ghisi D, et al. Intrathecal 1\% 2-chloroprocaine vs $0.5 \%$ bupivacaine in ambulatory surgery: a prospective, observerblinded, andomized, controlled trial. Acta Anaesthesiol Scand. 2014; 58(5):560-566.

\section{Publish your work in this journal}

Ambulatory Anesthesia is an international, peer reviewed, open access journal publishing articles that address all aspects of ambulatory anesthesia practice, in particular: anesthetic techniques, sedation and safety practices, pharmacokinetics, preoperative evaluation, analgesia interventions, regulatory and compliance issues, postoperative recovery,

Submit your manuscript here: http://www.dovepress.com/ambulatory-anesthesia-journal

\section{Dovepress}

patient satisfaction, administrative topics, and cost analysis themes. The manuscript management system is completely online and includes a very quick and fair peer review system, which is all easy to use. Visit http://www.dovepress.com/testimonials.php to read real quotes from published authors. 